\title{
Strain hardening in bidisperse polymer glasses: Separating the roles of chain orientation and interchain entanglement
}

\author{
Robert S. Hoy \\ Materials Research Laboratory, University of California, Santa Barbara, CA 93106 \\ Mark O. Robbins \\ Department of Physics and Astronomy, Johns Hopkins University, Baltimore, MD 21218
}

(Dated: November 24, 2018)

\begin{abstract}
The effects of entanglement and chain orientation on strain hardening in glassy polymers are separated by examining mixtures of chains with different lengths. Simulations show that the orientation of a molecule of a given chain length is the same in monodisperse systems and bidisperse mixtures, even when entangled and unentangled chains are mixed. In addition, the stress in mixtures is equal to the weighted average of the stresses in monodisperse systems. These results indicate that chains contribute independently to strain hardening, that chain orientation is determined by local interactions with the surrounding glass, and that entanglements play at most an indirect role in strain hardening in the range of strains typically studied. We discuss these results in the context of recent microscopic theories.
\end{abstract}

PACS numbers: 61.41.+e,62.20.F-,81.40.Lm,83.10.Rs

\section{INTRODUCTION}

The mechanical performance and failure modes of glassy polymers are strongly affected by strain hardening; an increase in the stress needed to deform systems as the strain increases. There has been great interest in understanding the mechanisms of strain hardening so that it can be predicted and optimized for applications. Recent studies have challenged the traditional view that entanglements between polymer chains control strain hardening, and suggest that orientation of individual chains plays the dominant role. This paper uses simulations of polymer mixtures to separate the roles of entanglements and orientation.

The most widely used [1, 2] theories of strain hardening are based on rubber elasticity. They assume that entanglements between polymer chains act like chemical crosslinks in a rubber and that strain hardening arises from the decrease in entropy of an affinely deforming entanglement network [3]. The stress is written as

$$
\sigma(\bar{\lambda}) \simeq \sigma_{\text {flow }}+G_{R} g(\bar{\lambda})
$$

where $\sigma_{\text {flow }}$ is the plastic flow stress, $G_{R}$ the "strain hardening modulus", $\bar{\lambda}$ the stretch tensor describing the macroscopic deformation, and $g$ a dimensionless function that describes the reduction in chain entropy. This approach has had much success in fitting experimental stress strain curves, but the fits seem inconsistent with the underlying microscopic model [4]. One difficulty is that $G_{R}$ is two orders of magnitude larger than expected near the glass transition temperature $T_{g}$. More troubling is that strain hardening grows with decreasing temperature, while any entropic force should decrease. A final

*Electronic address: robert.hoy@yale.edu difficulty is that the theory does not explain the plastic flow stress, which must be added by hand.

A number of recent experiments and simulations have examined the origins of these difficulties $[4,5,6,6,7,8,9$, $10,11,12,13,14,15,16,17$. One realization is that the two terms on the right hand side of Eq. (11) are of similar magnitude and must arise from similar mechanisms. Experiment, theory and simulations all show that $\sigma_{\text {flow }}$ and $G_{R}$ are linearly related when pressure, $T$ or strain rate is varied [10, 15, 16, 18]. This connection explains the large magnitude of $G_{R}$ and the fact that it grows with decreasing temperature as is typical for the plastic flow stress. A direct connection between $\sigma_{\text {flow }}$ and $G_{R}$ has been established in the athermal limit $(T \rightarrow 0)$, where both contributions to the stress are given by a single scaling factor times the rate at which interchain bonds break during local plastic rearrangements 12, 13.

Simulations also show strain hardening occurs in polymers that are too short to form the entangled network that is assumed in rubber elasticity theories $[12,13,14$, 17]. Moreover, the strain hardening in these unentangled systems can be mapped to that of entangled chains if the macroscopic deformation $\bar{\lambda}$ in Eq. 1 is replaced [12, 13] by an effective stretch tensor $\bar{\lambda}_{\text {eff }}$ that describes the orientation of individual chains:

$$
\sigma(\bar{\lambda})=\sigma_{\text {flow }}+G_{R}^{0} g\left(\bar{\lambda}_{\text {eff }}\right),
$$

where $G_{R}^{0}$ is the value of $G_{R}$ in the long-chain limit. The orientation of entangled chains is consistent with an affine deformation, $\bar{\lambda}_{e f f}=\bar{\lambda}$. For unentangled systems the orientation is consistent with a subaffine deformation by $\bar{\lambda}_{e f f}$.

The above findings support the notion that entanglements play an indirect role and that strain hardening is produced by the increased rate of local plastic rearrangements that are needed to maintain chain connectivity as 
chains orient 12, 13, 19]. In contrast, other recent experiments [5, 20] suggest that entanglements play a central role. They find that $G_{R}$ is directly proportional to the entanglement density $\rho_{e}$ in both pure systems and polymer mixtures. However, there are other explanations for this correlation. For example, an increase in chain stiffness increases $\rho_{e}$ and also stretches the chains so that more plastic rearrangements are required to maintain chain connectivity $13,17,21,22$.

In this paper we examine strain hardening of polymer mixtures. Using polymers with different chain lengths but the same interactions, stiffness, etc., allows us to isolate the roles of entanglements and orientation. The simulation results show that the orientation of chains plays the dominant role and that the response of mixtures has a surprisingly simple mean-field form. The variation of chain orientation with macroscopic stretch is nearly independent of the length of surrounding chains. In addition, the stress in mixtures is a simple weighted average of results for pure systems. These results suggest that strain hardening can be modeled as a sum of contributions from individual chains as they are deformed by interactions with the surrounding glass and produce local plastic rearrangements in the glass to maintain their connectivity.

\section{POLYMER MODEL AND METHODS}

Molecular dynamics (MD) simulations are performed using a coarse-grained bead-spring polymer model [23]. All monomers have mass $m$ and interact via the truncated and shifted Lennard-Jones potential

$$
U_{L J}(r)=4 u_{0}\left[(a / r)^{12}-(a / r)^{6}-\left(a / r_{c}\right)^{12}+\left(a / r_{c}\right)^{6}\right],
$$

where $r_{c}=1.5 a$ is the cutoff radius and $U_{L J}(r)=0$ for $r>r_{c}$. Covalent bonds between adjacent monomers on a chain are modeled using the FENE potential

$$
U(r)=-0.5 k R_{0}^{2} \ln \left(1-\left(r / R_{0}\right)^{2}\right),
$$

with the canonical [23] parameter choices $R_{0}=1.5 a$ and $k=30 u_{0} / a^{2}$. The equilibrium covalent bond length is $l_{0}=0.96 a$. We consider semiflexible chains with an angular potential $U_{\text {bend }}=k_{\text {bend }}(1-\cos (\theta))$, where $\theta$ is the angle between consecutive bond vectors along a chain. All quantities are expressed in units of the molecular diameter $a$, binding energy $u_{0}$, and characteristic time $\tau_{L J}=\sqrt{m a^{2} / u_{0}}$. Periodic boundary conditions are imposed, with periods $L_{i}$ along directions $i=x, y$, and $z$.

Simulations are performed using the same protocols as in Refs. 11, 12, 13]. A rapid quench is applied to well-equilibrated melts [24], producing glasses at the desired temperature $T$. The rapidity of the quench suppresses aging and strain softening. The simulations presented here are performed at $T=0.2 u_{0} / k_{B}$ and $T=$ $0.275 u_{0} / k_{B}$. Both values are well below the glass transition temperature $T_{g} \simeq u_{0} / 3 k_{B}$ [25], yet sufficiently high to observe significant thermally-activated rate-dependent relaxation [11]. Uniaxial compression is performed at constant strain rate $\dot{\epsilon} \equiv \dot{L}_{z} / L_{z}$. The volume stays nearly constant during compression. In this case the function describing the entropy loss in Eq. 1 can be written as $g(\lambda)=1 / \lambda^{2}-\lambda$ where $\lambda=L_{z} / L_{z}^{0}$ is the stretch relative to the initial size $L_{z 0}$.

The strain rates used range from $10^{-6} / \tau_{L J}$ to $10^{-4} / \tau_{L J}$. While these rates are higher than experiment, previous studies in this range of $\dot{\epsilon}$ and $T$ have been shown to capture many aspects of experiments, including logarithmic rate dependence [25], creep [26], and a linear relationship between flow and hardening modulus [15]. Our rates are also orders of magnitude slower than those obtainable with more computationally intensive united atom models (e. g. Ref. [17]). At the higher rates studied with these potentials, unentangled chains deform nearly affinely because there is insufficient time for activated motion of chains relative to their neighbors. This motion is particularly important for the chain length dependence of strain hardening considered here.

Systems contain short and long chains with $N_{\text {short }}$ and $N_{\text {long }}$ monomers, respectively. The total number of monomers is $N_{t o t}$ and the weight fraction of short chains is $f$. We present results for $N_{\text {long }}=350,10 \leq$ $N_{\text {short }} \leq 25$, and $k_{\text {bend }}=0.75 \epsilon$, but equivalent results were obtained for a range of other values. For this $k_{\text {bend }}$, the entanglement length is $N_{e} \sim 40$ monomers [27]. In experiments, unentangled glasses with $N \lesssim N_{e}$ typically exhibit brittle fracture, but fracture is suppressed in simulations [11, 14], perhaps because of the small system size and periodic boundary conditions.

\section{RESULTS}

Panels (a) and (b) of Figure 1 show the stress as a function of $g(\lambda)$ for mixtures with $N_{\text {short }}=10$ and 25, respectively. Results for $f=0.5$ lie directly between the results for pure systems with $f=0$ and 1 . Also shown is the weighted average of the pure system results

$$
\sigma_{\text {ave }}=(1-f) \sigma_{\text {long }}+f \sigma_{\text {short }}
$$

for $f=0.5$. Within our statistical accuracy, the stress in the mixtures is equal to this weighted average. We have verified that this "stress superposition principle" holds for different $k_{\text {bend }}$, chain lengths, temperatures and $f$. The only discrepancies are observed at large $g$ $(>3.5)$ when chains in entangled systems have stretched nearly taut and significant energy is stored in the covalent bonds. Experiments only reach such large stretches when special sample preparation procedures are used [2, 29].

Equation (1) would predict a linear increase in stress with $g$. The systems in Fig. 1 were chosen to exhibit deviations from this straight line behavior, but still obey stress superposition. Deviations from linearity in experiments are often fit by including non-Gaussian (finite chain length) corrections to the network entropy 

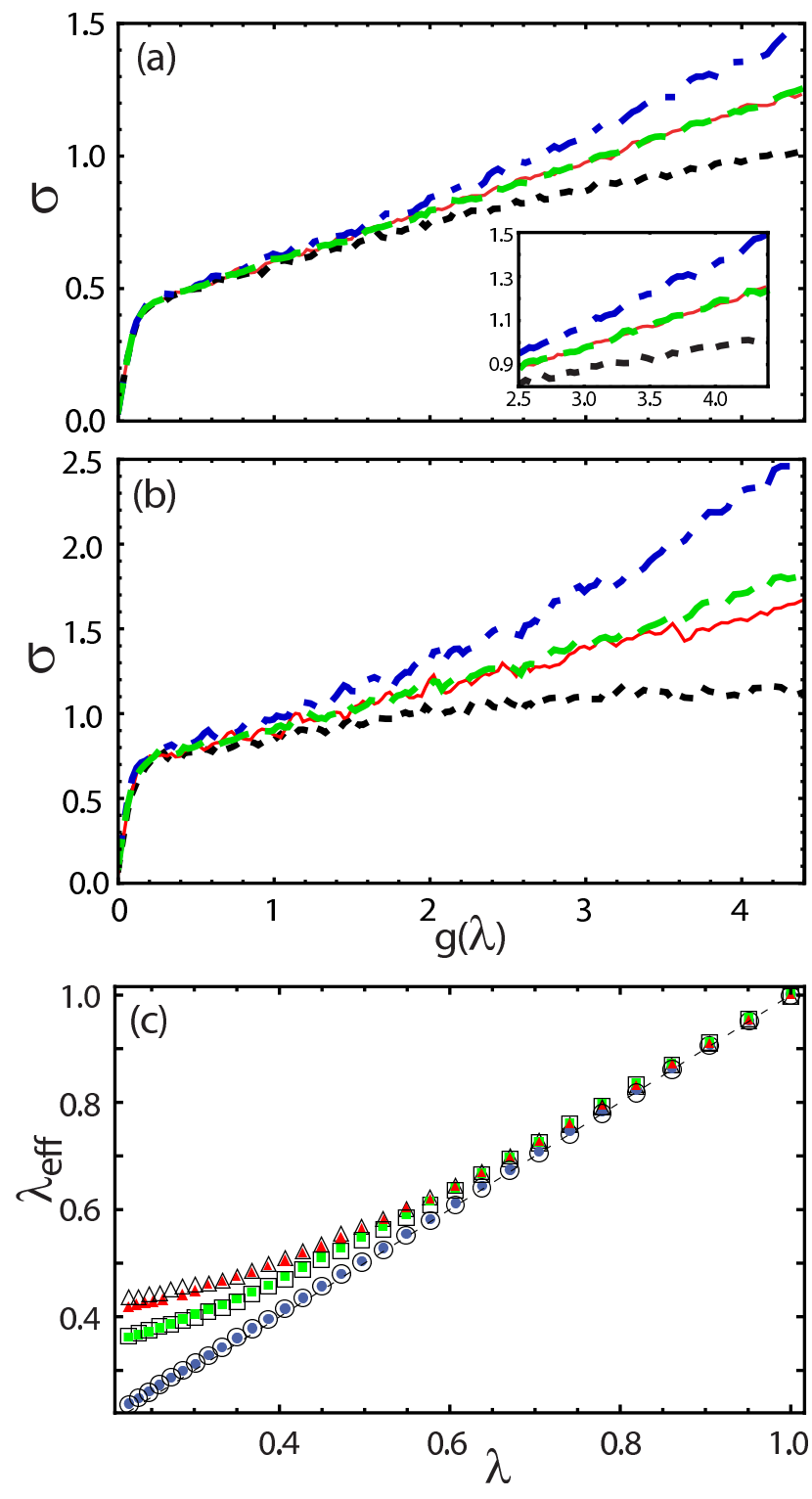

FIG. 1: Panels (a-b): Stress plotted against $g(\lambda)$ for uniaxial compression of monodisperse glasses with length $N_{\text {long }}$ (dashdotted) or $N_{\text {short }}$ (dotted) and of 50/50 mixtures of the two lengths (dashed). The solid curves show the average of the two monodisperse systems. Here $k_{\text {bend }}=0.75\left(N_{e}=39\right)$. Panel (a): $N_{\text {short }}=25, T=.275 u_{0} / k_{B}$ and $|\dot{\epsilon}|=10^{-5} / \tau_{L J}$. The inset shows a blowup of the data at large $g$ [28]. Panel (b): $N_{\text {short }}=10, T=0.2 u_{0} / k_{B}$ and $|\dot{\epsilon}|=10^{-4} / \tau_{L J}$. Panel (c): Variation of $\lambda_{\text {eff }}$ with $\lambda$ for pure systems with $N=350$ (solid circles), $N=25$ (solid squares), and $N=10$ (solid triangles). Open symbols of the same shape show results for chains of each length in $f=0.5$ mixtures.

[2]. Previous simulations are not consistent with this interpretation [12, 13]. They show that the upturn in stress for entangled systems reflects energy stored in the glass, while the downward curvature for unentangled systems results from subaffine deformation of the chains $\left.\left(\mid \ln \left(\lambda_{e f f}\right)\right)|<| \ln (\lambda) \mid\right)$. Despite the different curvatures for the long and short chains in Fig. 1 their contributions to the stress in mixtures are simply additive.

These results do not conflict with experiments that found $G_{R}$ was proportional to $\rho_{e}[\underline{5},[30]$. For the specific polymers studied, the entanglement density of mixtures is a weighted average of pure systems. Thus these systems also obey stress superposition. It would be interesting to perform similar experiments on polymer mixtures whose entanglement density was a nonlinear function of weight fraction.

The relation between $\rho_{e}$ and $f$ is nonlinear for the model polymers considered here, yet plots in a previous paper indicated $G_{R}$ was proportional to $\rho_{e}$ [11]. While this is approximately true for monodisperse systems, the results for mixtures were misinterpreted in this earlier paper. A factor of $(1-f)$ was inadvertently excluded in the calculation [31] of $\rho_{e}$ from Primitive Path Analysis [27]. When $\rho_{e}$ is evaluated correctly, the hardening modulus is no longer proportional to $\rho_{e}$. However, we do find that the values of $G_{r}$ from Ref. [11] satisfy stress superposition. Indeed, the superposition principle explains the success of the previous collapse. As noted in Ref. [11], the incorrectly determined value of $\rho_{e}$ was proportional to $(1-f)$ and so the collapse implied $G_{R} \propto(1-f)$ as expected from superposition. The correct $\rho_{e}$ is nearly proportional to $(1-f)^{2}$ and not linearly related to $G_{R}$.

To understand why the contribution of chains to stress is additive, we examined the changes in molecular conformations during shear. As in Ref. [12], the orientation was quantified by the effective stretch $\lambda_{e f f}$. This is defined as the mean stretch of chains along the compression direction; $\lambda_{\text {eff }}=\left\langle R_{z} / R_{z}^{0}\right\rangle$, where $R_{z}$ is the rms $z$-component of the end-to-end vector of a chain and $R_{z}^{0}$ the value before strain. Panel (c) shows $\lambda_{\text {eff }}(\lambda)$ for short and long chains in the pure and mixed states for the same systems shown in panels (a-b). Remarkably, $\lambda_{\text {eff }}^{\text {long }}$ and $\lambda_{\text {eff }}^{\text {short }}$ do not themselves depend on $f$. In other words, the orientation of the long (short) chains is not dependent on whether they are in a pure system or mixed with a fairly high fraction of short (long) chains. This $f$-independence of $\lambda_{e f f}$ holds over the same broad range of conditions as the stress superposition principle.

How can we understand the apparent $f$-independence of $\lambda_{e f f}$ ? The simplest qualitative picture is that interchain interactions in the glass tend to enforce an affine deformation of the chains, but that this is opposed by intrachain bonds. Chains can not deform affinely at small scales because this would stretch the stiff covalent bonds along the chains. Instead, chains are observed to straighten over an effective persistence length $l_{p}$ that increases with $g$ [11, 17]. This straightening is independent of chain length until the associated stress becomes large enough to force relaxation of the chain along its length. For the systems considered here, the friction with neighboring chains is large enough that significant relaxation does not occur until unentangled chains are stretched to a substantial fraction of their fully extended length. Straightening chains while maintaining their con- 
nectivity requires local rearrangements of the surrounding monomers. The number of rearrangements grows with the persistence length and this was found to correlate directly with the strain hardening at low temperatures [12, 13, 17, 21].

Note that entanglements do not enter directly in this picture. Since entangled and unentangled chains behave in the same manner at small $|g|$, the constraints on their rearrangements must be determined by very local interactions with the surrounding matrix. Entanglements only become relevant when $g$ is so large that $l_{p}$ reaches a significant fraction of the separation between entanglements [12, 13]. In this limit entanglements become more effective than friction from the matrix in enforcing an affine displacement of the chain. The associated tension in the chains grows as $l_{p}$ approaches $N_{e} l_{0}$ and contributes significant energetic terms to the strain hardening at large $|g|$ [13, 29, 32, 33].

It is worth considering under which conditions this mean field picture should break down. For the reasons just described, the range of validity will be limited to small strains in very densely entangled systems; fortunately, by this standard, few synthetic polymers are so densely entangled [34]. The mean field picture should also break down for extremely short chains where the high density of chain ends changes the density, friction and other properties significantly. Stress superposition will be particularly sensitive to changes in the relaxation rate of chains on the end-end scale, $\tau_{\text {relax }}^{-1}$, with $f$. These are known to be significant for $T$ above $T_{g}$, where $\tau_{\text {relax }}$ for short chains is increased when they are mixed with longer chains, leading to greater orientation at a given shear rate 35,36$]$. Our results show that such coupledorientation effects are small deep in the glassy state.

\section{DISCUSSION AND CONCLUSIONS}

The simulations presented here provide further evidence that strain hardening in polymer glasses depends primarily on chain orientation rather than entanglement. They also provide new insight into the factors that control the degree of orientation. The local coupling to the glassy matrix enforces an affine deformation of the chains $\left(\lambda=\lambda_{e f f}\right)$ at small $|g|$. Hardening arises primarily from the plastic rearrangements needed to maintain intrachain connectivity as chains orient and are straightened over greater lengths. As the associated stress on the chains grows, there is an increasing tendency for them to relax along their length, leading to subaffine deformation $\left(\lambda \neq \lambda_{e f f}\right)$. The observation that the value of $\lambda_{e f f}$ for a given chain length is independent of $f$ and that the stress due to plastic rearrangements obeys the superposition principle, implies that chains relax nearly independently. The degree of relaxation depends on a competition between the intrachain tension caused by alignment and friction with the glassy matrix. Entanglements only enter at very large $|g|$ where they prevent relaxation of chains that are much longer than $N_{e}$.

To our knowledge, no microscopic theory that predicts the functional form of $\lambda_{e f f}$ in glasses has been published. Constitutive models which employ a viscoelastic or viscoplastic description of glassy strain hardening (e. g. Refs. [37, 38, 39]) typically decompose $\bar{\lambda}$ into rubberelastic and plastic parts (or use other internal state variables), but do not explicitly account for $\lambda \neq \lambda_{\text {eff }}$ or the $N$-dependence of nonaffine relaxation [40]. It would be interesting to see if a first principles theory like that of Ref. [18] could be generalized to predict $\lambda_{\text {eff }}(\lambda)$. The qualitative ingredients that must enter such a microscopic theory are evident from the results shown here and in Refs. [12, 13, 17]. Simply put, $\lambda_{e f f}$ falls behind $\lambda$ when the stress in the material is insufficient to further affinely orient the average chain.

Fortuitously, $\lambda_{e f f}$ can now be accurately measured in scanning near-field optical microscopy experiments [41] which can discriminate values for different chain lengths in a bidisperse system. Ref. [41] showed $\lambda_{\text {eff }}$ falls behind $\lambda$ for entangled chains deformed slightly above $T_{g}$; similar studies on a variety of chain lengths below $T_{g}$ would be of great interest. These might prove that $\lambda_{\text {eff }}$ is one of the "mesoscale" quantities [42] whose understanding will improve modeling of the deformation of amorphous materials. Other recent experiments [43] have shown that segmental dynamics and larger-scale polymer dynamics are nontrivially coupled below $T_{g}$. Further developments along these lines would be welcome, as would extension of the recently improved understanding of the relation [44] between linear viscoelastic behavior and structural relaxation to the nonlinear regime.

\section{Acknowledgments}

Kenneth S. Schweizer provided the original concept of $\lambda_{e f f}$. Daniel J. Read provided extensive, helpful correspondence regarding mixtures, and Edward J. Kramer, Hugh R. Brown and Kang Chen provided helpful discussions. All simulations were performed using the LAMMPS MD code [45]. Gary S. Grest provided some equilibrated melt states. This material is based upon work supported by the National Science Foundation under Grant No. DMR-0454947 and through the MRSEC Program under Award No. DMR05-20415.
[1] R. N. Haward and G. Thackray, Proc. Roy. Soc. Lond. 302, 453 (1968).
[2] E. M. Arruda and M. C. Boyce, Int. J. Plast. 9, 697 (1993). 
[3] Hereon tensor notation for $\bar{\lambda}$ is dropped for convenience, but $\lambda$ and $\lambda_{\text {eff }}$ remain tensorial.

[4] E. J. Kramer, J. Polym. Sci. Part B - Polym. Phys. 43, 3369 (2005).

[5] H. G. H. van Melick, L. E. Govaert, and H. E. H. Meijer, Polymer 44, 2493 (2003).

[6] R. B. Dupaix and M. C. Boyce, Polymer 46, 4827 (2005).

[7] M. Wendlandt, T. A. Tervoort, and U. W. Suter, Polymer 46, 11786 (2005). M. Wendlandt, T. A. Tervoort, J. D. van Beek, and U. W. Suter, J. Mech. Phys. Solids 54, 589 (2006).

[8] P. J. Hine, A. Duckett, and D. J. Read, Macromolecules 40, 2782 (2007).

[9] F. Casas, C. Alba-Simionesco, H. Montes, and F. Lequeux, Macromolecules 41, 860 (2008).

[10] L. E. Govaert, T. A. P. Engels, M. Wendlandt, T. T. A, and U. W. Suter, J. Polym. Sci. Part B - Polym. Phys. 46, 2475 (2008).

[11] R. S. Hoy and M. O. Robbins, J. Polym. Sci. Part B Polymer Phys. 44, 3487 (2006).

[12] R. S. Hoy and M. O. Robbins, Phys. Rev. Lett. 99, 117801 (2007).

[13] R. S. Hoy and M. O. Robbins, Phys. Rev. E 77, 031801 (2008).

[14] A. V. Lyulin, N. K. Balabaev, M. A. Mazo, and M. A. J. Michels, Macromolecules 37, 8785 (2004); A. V. Lyulin, B. Vorselaars, M. A. Mazo, N. K. Balabaev, and M. A. J. Michels, Europhys. Lett, 71, 618 (2005).

[15] M. O. Robbins and R. S. Hoy, J. Polym. Sci. Part B Polym. Phys. 47, 1406 (2009).

[16] B. Vorselaars, A. V. Lyulin, and M. A. J. Michels, J. Chem. Phys. 130, 074905 (2009).

[17] B. Vorselaars, A. V. Lyulin, and M. A. J. Michels, Macromolecules 42, 5829 (2009).

[18] K. Chen and K. S. Schweizer, Phys. Rev. Lett 102, 038301 (2009).

[19] A. S. Argon, Philos. Mag. 28, 39 (1973).

[20] C. G'Sell and A. Souahi, J. Eng. Materials Tech. 119, 223 (1997).

[21] J. I. Mckechnie, R. N. Haward, D. Brown, and J. H. R. Clarke, Macromolecules 26, 198 (1993).

[22] L. J. Fetters, D. J. Lohse, D. Richter, T. A. Witten, and A. Zirkel, Macromolecules 27, 4639 (1994).

[23] K. Kremer and G. S. Grest, J. Chem. Phys. 92, 5057 (1990).

[24] R. Auhl, R. Everarers, G. S. Grest, K. Kremer, and S. J. Plimpton, J. Chem. Phys. 119, 12718 (2003).

[25] J. Rottler and M. O. Robbins, Phys. Rev. E 68, 011507 (2003).
[26] M. Warren and J. Rottler, Phys. Rev. E 76, 031802 (2007); R. A. Riggleman, K. S. Schweizer, and J. J. de Pablo, Macromolecules 41, 4969 (2008).

[27] R. Everaers, S. K. Sukumaran, G. S. Grest, C. Svaneborg, A. Sivasubramanian, and K. Kremer, Science 303, 823 (2004).

[28] A smaller system with the same parameters as in panel (a) was tested at $|\dot{\epsilon}|=10^{-6} / \tau_{L J}$ and gave equivalent results, but these were noisier due to finite size effects. Changing the rate by two orders of magnitude had only a modest effect on $\lambda_{e f f}$, as expected from a logarithmic rate sensitivity.

[29] O. A. Hasan and M. C. Boyce, Polymer 34, 5085 (1993).

[30] Z. Bartczak, Macromolecules 38, 7702 (2005).

[31] We thank Alexei E. Likhtman and Daniel J. Read for pointing out an inconsistency that led to this discovery.

[32] C. Chui and M. C. Boyce, Macromolecules 32, 3795 (1999).

[33] J. Li, T. Mulder, B. Vorselaars, A. V. Lyulin, and M. A. . J. Michels, Macromolecules 39, 7774 (2006).

[34] R. N. Haward, Macromolecules 26, 5860 (1993).

[35] A. R. C. Baljon, G. S. Grest, and T. A. Witten, Macromolecules 28, 1835 (1995).

[36] C. M. Ylitalo, J. A. Kornfield, G. G. Fuller, and D. S. Pearson, Macromolecules 24, 749 (1991).

[37] L. Anand and M. E. Gurtin, Int. J. Solids Structures 40, 1465 (2003).

[38] J. M. Caruthers, D. B. Adolf, R. S. Chambers, and P. Shrikhande, Polymer 45, 4577 (2004).

[39] R. B. Dupaix and M. C. Boyce, Mech. Materials 39, 39 (2007).

[40] Ref. 46] considered $N$-dependent (reptational) relaxation of incompletely crosslinked elastomers above $T_{g}$ and it would be interesting to see if this approach could be adapted to glassy systems.

[41] T. Ube, H. Aoki, S. Ito, J. Horinaka, and T. Takigawa, Polymer 48, 6221 (2007); T. Ube, H. Aoki, S. Ito, J. Horinaka, T. Takigawa, and T. Masuda, Polymer 50, 3016 (2009).

[42] J. Barrat and J. J. de Pablo, MRS Bull. 32, 941 (2007).

[43] J. Hintermeyer, A. Herrmann, R. Kahlau, C. Goiceanu, and E. A. Rössler, Macromolecules 41, 9335 (2008).

[44] L. Grassia and A. D'Amore, J. Polym. Sci. Part B Polym. Phys. 47, 724 (2009).

[45] http://lammps.sandia.gov/.

[46] J. S. Bergström and M. C. Boyce, J. Mech. Phys. Solids 46, 931 (1998). 